\title{
Could People with Stereo-Deficiencies Have a Rich 3D Experience Using HMDs?
}

\author{
Sonia Cárdenas-Delgado ${ }^{1}$, M.-Carmen Juan ${ }^{1(\bowtie)}$, \\ Magdalena Méndez-López ${ }^{2}$, and Elena Pérez-Hernández ${ }^{3}$ \\ 1 Instituto Universitario de Automática e Informática Industrial, \\ Universitat Politècnica de València, Camino de Vera, s/n, 46022 València, Spain \\ \{scardenas, mcarmen\}@dsic.upv.es \\ 2 Departamento de Psicología y Sociología, \\ Universidad de Zaragoza, Saragossa, Spain \\ mmendez@unizar.es \\ 3 Departamento de Psicología Evolutiva y de la Educación, \\ Universidad Autónoma de Madrid, Madrid, Spain \\ elena.perezh@uam.es
}

\begin{abstract}
People with stereo-deficiencies usually have problems for the perception of depth using stereo devices. This paper presents a study that involves participants who did not have stereopsis and participants who had stereopsis. The two groups of participants were exposed to a maze navigation task in a 3D environment in two conditions, using a HMD and a large stereo screen. Fifty-nine adults participated in our study. From the results, there were no statistically significant differences for the performance on the task between the participants with stereopsis and those without stereopsis. We found statistically significant differences between the two conditions in favor of the HMD for the two groups of participants. The participants who did not have stereopsis and could not perceive 3D when looking at the Lang 1 Stereotest did have the illusion of depth perception using the HMD. The study suggests that for the people who did not have stereopsis, the head tracking largely influences the $3 \mathrm{D}$ experience.
\end{abstract}

Keywords: HMD $\cdot$ Large stereo screen $\cdot$ Virtual reality $\cdot$ Stereopsis $\cdot 3 D$ experience $\cdot$ Stereoblindness $\cdot$ Stereo-deficiency $\cdot$ Head tracking

\section{Introduction}

Stereopsis refers to the perception of depth through visual information that is obtained from the two eyes of an individual with normally developed binocular vision [1]. The perception of depth is also possible with information visible from only one eye. In this case, the person uses differences in object size and motion parallax in order to have such perception [2]. However, according to Barry [3], the impression of depth cannot be as vivid as that obtained from binocular disparities.

Virtual Reality (VR) has received numerous definitions. According to LaViola [4], "VR is a user interface technology that provides an immersive and realistic, three dimensional computer simulated world". Dionisio et al. [5] defined VR as a 
computer-generated simulation of three-dimensional objects or environments with seemingly real, direct, or physical user interaction. Different devices or systems can be used to display a virtual environment. Different taxonomies have been established according to the level of immersion. For example, Muhanna [6] classified the VR systems as:

- Basic: hand-based and monitor-based.

- Partially immersive: wall projectors, immersive-desk, and monocular head-based.

- Fully immersive: room-based (vehicle simulation and CAVE) and binocular head-based.

In a subject of Virtual and Augmented Reality of a Master's program in Computer Science, we observed that students who did not have stereopsis (checked using the Lang 1 Stereotest) did not have perception of depth when using VR devices such as a CAVE, a large stereo screen and even with autostereoscopic displays. However, the same students had the sensation of depth using the Oculus Rift. This motivated us to design a study to compare the Oculus Rift with another stereo device that we have already used in order to test our hypothesis. The visualization system chosen for the comparison was a large stereo screen. However, other Head-Mounted Displays (HMD) or different visualization systems (CAVE, autostereoscopic displays, or other HMDs) could also be used. Since the appearance of the first HMD developed by Sutherland [7], many different commercial devices and non-commercial prototypes have been developed (e.g., Oculus Rift, HTC VIVE, Google Cardboard, Samsung Gear VR, or Microsoft HoloLens). Therefore, other comparisons could be considered in the future work.

In this paper, we present a study in which users were exposed to a maze navigation task in a 3D environment in two conditions: using a HMD (Oculus Rift) and using polarization glasses with a large stereo screen. Two groups of users participated in our study: a group who did not have stereopsis (no stereopsis), and a group who had stereopsis (stereopsis). Our main hypothesis was that the users that did not have stereopsis would have a statistically richer 3D experience with the HMD than with a large stereo screen.

\section{Background}

\subsection{Stereopsis Recovery}

Several previous works have focused on the idea of restoring stereopsis in adults. Two cases in which this recovering was described were experienced by Barry [3] and Bridgeman [8]. Barry [3] recovered from strabismus after visual therapy in adulthood. Bridgeman [8], with stereo-deficiency, acquired stereopsis when watching a 3D movie. Besides these two personal experiences, other works have also been interested in stereopsis recovery. For example, Ding \& Levi [9] carried out a case study involving 5 adults who were stereoblind or stereoanomalous. After perceptual learning, the participants showed substantial recovery of stereopsis. Ding \& Levi [9] concluded that "some human adults deprived of normal binocular vision can recover stereopsis at least 
partially". In the same year, Astle et al. [10] carried out another case study involving two humans with anisometropic amblyopia whose stereopsis also improved after following a training course. In 2014, Xi et al. [11] carried out a case study involving 11 participants with anisometropic or ametropic amblyopia. Those participants were trained with anaglyphic textures with different disparities. They also experienced stereopsis improvement. Vedamurthy et al. [12] trained adults who were stereo blind or stereodeficient using a natural visuomotor task (a Virtual Reality environment). They conclude that "some adults deprived of normal binocular vision and insensitive to the disparity information can, with appropriate experience, recover access to more reliable stereoscopic information". Therefore, all these previous works indicate that human adults can recover or acquire stereopsis in adulthood.

\subsection{Users' Perceptions}

In this section, we focus on users' perceptions in which two different visualization devices have been compared. To our knowledge, no previous work has studied users' perceptions considering people with stereo vision versus stereo blindness. However, several studies of users' perceptions have been carried out with groups in which specific problems had not been defined.

With regard to the comparison between different HMDs, Young et al. [13] compared the Oculus Rift and a high-cost Nvis SX60 HMD, which differ in resolution, field of view, and inertial properties, among other factors. In this comparison, both HMDs were fully immersive. Young et al. [13] assessed simulator sickness and presence. They found that the Oculus Rift consistently outperformed the Nvis SX60 HMD, but some people were more subject to simulator sickness with the Oculus Rift. Bun et al. [14] used the nVisor MH60 V HMD, the Oculus Rift DK1, and Samsung Gear VR with students of medical disciplines to learn anatomy. In this study, the three HMDs were fully immersive. Twenty students from the Poznan University of Technology participated in a study concerning perception. The participants were asked to select the preferred HMD and interaction method. Most of them chose the Gear VR in combination with Kinect and gamepad as the preferred solution.

Other works have compared HMDs with different visualization systems. For example, Tan et al. [15] presented a study involving 10 participants who played a first-person shooter game using the Oculus Rift and a traditional desktop computer-monitor. In that study, the authors compared a fully immersive VR system with a basic or low immersive VR system. They concluded that the participants had heightened experiences, a richer engagement with passive game elements, a higher degree of flow, and a deeper immersion with the Oculus Rift than on a traditional desktop computer-monitor. However, they also mentioned the problems of cybersickness and lack of control. Gutiérrez-Maldonado et al. [16] developed a VR system to train diagnostic skills for eating disorders and compared two visualization systems (Oculus Rift DK1 vs. a laptop with a stereoscopic 15.6-inch screen). In that study, the authors also compared a fully immersive VR system with a basic or low immersive VR system. Fifty-two undergraduate students participated in their study. No differences were found in either effectiveness or usability with regard to skills training in a psychopathological exploration of eating disorders through virtual simulations. 
Juan \& Pérez [17] carried out a comparison study of the levels of presence and anxiety in an acrophobic environment that was viewed using a Computer Automatic Virtual Environment (CAVE) and a 5DT HMD. In this environment, the floor fell away, and the walls rose up. To determine whether either of the two visualization systems induced a greater sense of presence/anxiety in non-phobic users, an experiment comparing the two visualization systems was carried out. Twenty-five non-phobic participants took part in their study. The CAVE induced a significantly higher level of presence in users. Their results indicated that both visualization systems provoked anxiety, but that the CAVE provoked more anxiety than the HMD.

Other works have compared different versions of the same environment using HMDs. For example, Davis et al. [18] used the Oculus Rift and compared two different virtual roller coasters, each with different levels of fidelity. They found that the more realistic roller coaster with higher levels of visual flow had a significantly greater chance of inducing cybersickness.

Therefore, previous works have compared HMDs with low and fully immersive VR systems. In contrast, we compare a HMD with a partially immersive VR system.

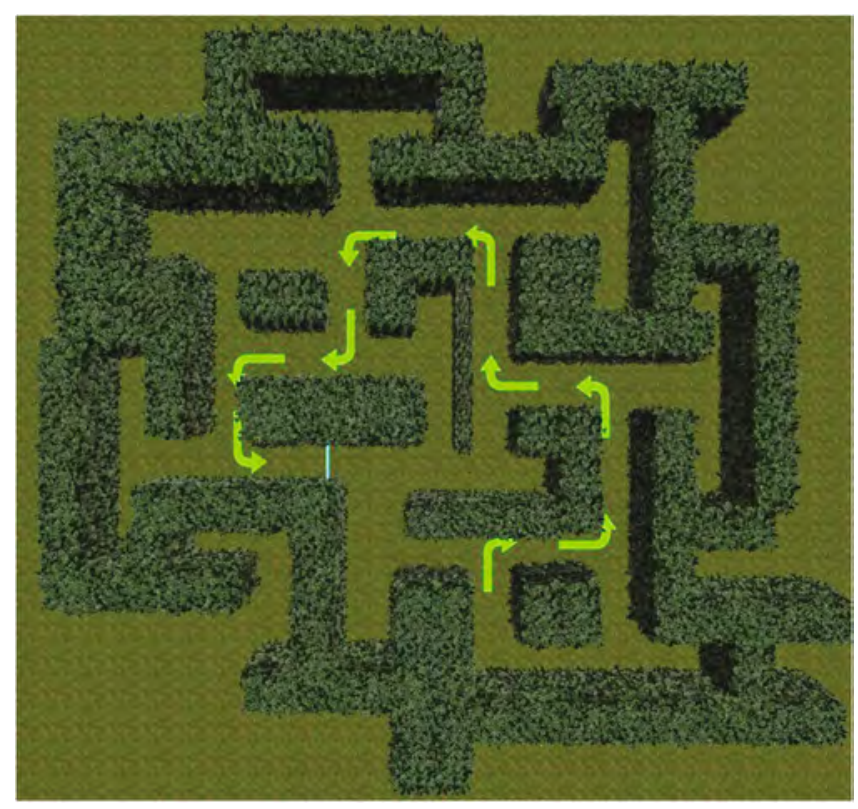

Fig. 1. Virtual environment. Maze viewed from above.

\section{Virtual Environment}

The virtual environment is based on the Cincinnati Water Maze [19]. The virtual environment is a maze with nine intersections (see Fig. 1). The maze has a wall of hedges that are two meters high and pathways of grass that are two meters wide. A first-person avatar represents the participant's point of view (the participant sees the 
maze through the eyes of the avatar). Therefore, this avatar personifies the movements of the user in the maze. The participant controls the avatar with a gamepad. At each intersection, there is an arrow on the ground; the color of the arrow and the direction it points depend on the scene. The system has three stages: habituation, retention, and testing. The habituation stage has an environment with a short route. The path has four intersections and a straight road at the end. This is a trial stage to train participants to handle the system properly. The retention stage consists of an environment in which the participant follows another route with nine intersections and is guided by green arrows. The participant must learn the route. The testing stage has yellow arrows that show options at each intersection. The participant must remember and follow the same path that was followed in the retention stage. When the participant makes a mistake, the system shows a warning message and they are automatically relocated back to the starting position. Each participant has five attempts to reach the end of the maze. The time increases with the number of attempts. The experience lasts around six minutes. However, the time could increase based on the number of attempts. A more detailed description of the virtual environment can be found in [20].

\subsection{Hardware and Software}

The virtual environment ran on an Intel Core i7 computer, $3.5 \mathrm{GHz}$ processor with 16 GB RAM, an NVIDIA GeForce GTX-970 with a video card of 4 GB, and Windows 8 Operating System. For the development of the system, we used Unity Edition Professional (http://unity3d.com), version 4.6.0f3 as the game engine, and C\# and JavaScript as the programming languages. Blender was used to create and modify the 3D models that were included in the environment. Adobe Photoshop was used to modify textures and images.

Two loudspeakers were used to provide messages and instructions to the participants. AB-Move Gamepad BG Revenge was used as the input device. Thanks to the gamepad, the user controlled the avatar, indicating the direction to follow (i.e., go forward, turn to the right, or turn to the left). The gamepad was integrated into the system thanks to the controller Input Manager of Unity, which enabled functions and personalized the use of the device in the two visualization systems. The collision of objects in the environment was controlled to keep the participants from colliding with the walls.

\subsection{Oculus Rift}

We used an Oculus Rift DK2. It has a resolution of $960 \times 1080$ per eye, a field-of-view of 100 nominal, a weight of $0.32 \mathrm{~kg}$, and an optical frame rate of $75 \mathrm{~Hz}$. It has head tracking and positional tracking. Figure 2 shows a view of the Maze with the Oculus Rift. Figure 3 shows a user handling the Oculus Rift.

The head tracking of the Oculus Rift was used to let the user to look around in the position where he/she was. To integrate the Oculus Rift with the system, we used the plugins provided by the manufacturer (Oculus SDK 0.4.2, Oculus Runtime, and Oculus Unity Integration Package). 


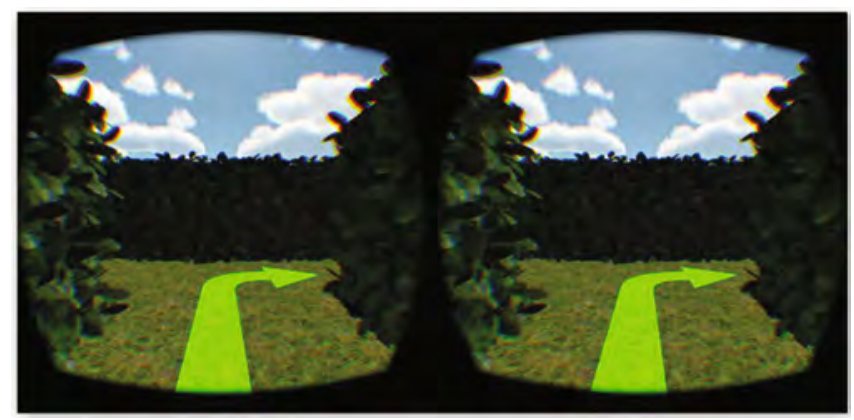

Fig. 2. View of the Maze with the Oculus Rift.

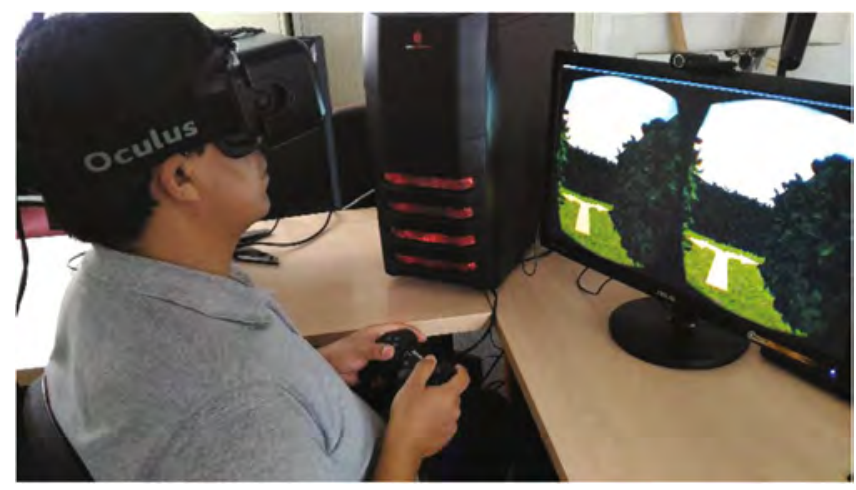

Fig. 3. A participant carrying out the task with the gamepad.

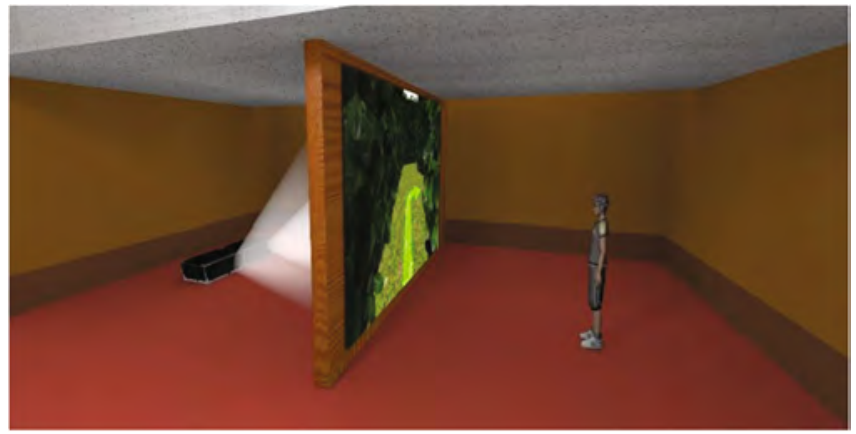

Fig. 4. Testing room for the large stereo screen condition.

\subsection{Large Stereo Screen}

A large stereo screen was placed in a room with some special characteristics. First, the room was divided into two areas (the projection area and the user area); these areas were separated by a wall and a translucent 120-inch screen. Figure 4 shows a 
representation of this room. Two projectors placed in the projection area project two images onto the screen. Specifically, we used two InFocus IN1503 short throw projectors. These projectors could generate an image of $177 \times 111 \mathrm{~cm}$. at a throw distance of $140 \mathrm{~cm}$. They produced a brightness of 3000 ANSI lumens and had a resolution of $1280 \times 800$ pixels. These two images are polarized and a 3D image is created. The user must wear linear polarized 3D glasses in order to see the image correctly. Figure 5 shows a user in the large stereo screen condition. A library was developed to create the $3 \mathrm{D}$ sensation. This library allows the user to have the right point of view by placing the two virtual cameras to simulate the two eyes of the user. The cameras are located at a standard intraocular distance $(63 \mathrm{~mm})$ [21] and at a field of view of $60^{\circ}$. This value for the field of view was calculated from the real dimensions of the screen and the distance between the participant and the screen. This condition did not include head tracking.

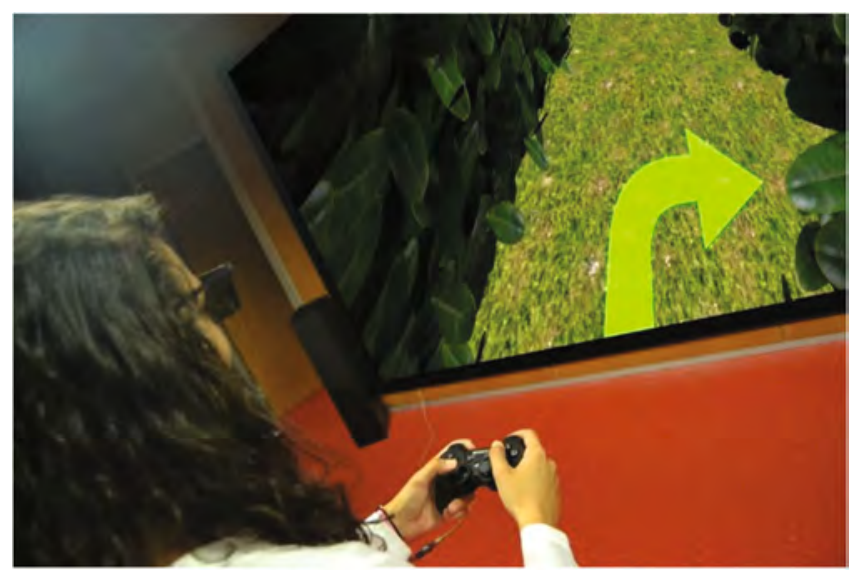

Fig. 5. A participant in front of the large stereo screen handling the gamepad.

\section{Study}

All of the participants were duly informed about the purpose of the study before each session. They signed the Informed Consent for participation, and the study was conducted according to the principles stated in the Declaration of Helsinki. The Ethics Committee of the Universitat Politècnica de València (UPV) approved this study.

Before each session, all of the participants filled out the Personal Data Questionnaire (PDQ). Afterwards, they were checked for stereopsis using the Lang 1 Stereotest cards $[22,23]$. The participants were divided into two groups. Each group participated in only one session and was exposed to only one of the two different conditions. One group used the HMD and the other group used the large stereo screen (intersubject analysis). Finally, when they had finished, they completed the questionnaire about previous experiences (PEQ), and another questionnaire (PQ) to know their perceptions about interaction, the 3D sensations, and satisfaction. Most of the questions of the PQ were adapted from the Presence Questionnaire proposed by Witmer \& Singer [24]. 


\subsection{Participants}

Students of the UPV participated in this study $(\mathrm{N}=59$; mean age $25.83 \pm 3.97$; 35 men and 24 women). A recruitment campaign was conducted to find the participants by advertising within the campus facilities. The participants were randomly assigned to each condition. Since the task was the same, each participant used only one of the two conditions. The participants were assigned randomly to two groups (30 participants for the HMD condition, and 29 for the large stereo screen condition).

\subsection{Control Variables}

To carry out the current study, two control variables were defined. The main goal was to establish homogeneous groups in terms of previous experiences with $3 \mathrm{D}$ and to determine which participants had stereopsis and which ones did not.

First, the PEQ was used to determine whether the participants of both groups had previous experience with 3D and video games. The PrevExperience variable combines the answers to questions related to previous experience (Table 1). The questionnaire used a Likert scale [from 1 to 5 (1 being 'none' or extremely low, and 5 being 'very high')]. For the group of participants who did not have stereopsis, the mean for the HMD condition was $3.09 \pm 0.61$, and the mean for the large stereo screen condition was $2.78 \pm 0.58$. These means indicate that those participants had moderate experience with 3D. For the group of participants who had stereopsis, the mean for the HMD condition was $3.10 \pm 0.59$, and the mean for the large stereo screen condition was $2.96 \pm 0.61$. These means indicate that those participants had moderate experience with 3D. There were no statistically significant differences in previous 3D experiences between the HMD condition and the large stereo screen condition $(U=34, Z=0.714$, $p=0.483, r=0.184)$. This result demonstrates the homogeneity of the sample regarding this aspect.

Table 1. Questionnaire on previous experience with 3D.

\begin{tabular}{l|l}
\hline$\# Q$ & Questions \\
\hline QX1 & I perform activities in 3D \\
\hline QX2 & I play 3D games \\
\hline QX3 & I see movies in 3D \\
\hline
\end{tabular}

Second, the Lang 1 Stereotest was applied to determine which participants had stereopsis and which ones did not. Brown et al. [23] administered the Lang 1 Stereotest to 292 participants and concluded that this test correctly identified people with vision defects associated with reduced stereopsis and that it was appropriate for vision screening of both adults and children. The Lang 1 Stereotest has three objects. We followed the protocol suggested by Brown et al. [23]. A participant passed the test when he/she had $3 / 3$ positive responses, $3 / 3$ partial positive responses, or $2 / 3$ positive and/or partial positive responses where the negative response was at the 550" level. A participant failed the test when he/she had $3 / 3$ negative responses and $2 / 3$ negative responses where the single positive or partial positive response was at the 1200 " level. 
In our sample, 22 participants were successful in the Lang 1 Stereotest for the HMD condition (73.33\%), and 22 participants were successful for the large stereo screen condition (75.86\%). Therefore, 8 participants failed the Lang 1 Stereotest in the HMD condition (26.67\%), and 7 participants failed in the large stereo screen condition $(24.14 \%)$. These results ensure an equivalent number of participants in the two conditions. In Brown et al.'s study [23], 6.5\% of the participants failed the test. Other studies have indicated that this percentage can be between 5\% and $10 \%$ [25], or as high as $34 \%$ in older subjects [26]. In our case, this percentage is considerably higher than in normal population. This is because we especially invited people who we knew did not have stereopsis to participate in our study. The objective was to have the sample of participants without stereopsis as large as possible to compare them with the population with stereopsis.

\subsection{Procedure}

The study compared the participant's perceptions using a 3D environment in two conditions (HMD and a large stereo screen). The same VR environment was used with each condition. In order to move around the virtual environment, the participants held a gamepad in their hands. In the HMD condition, the participants were seated in a chair and wore an Oculus Rift DK2 with head tracking enabled. The lenses of the HMD were positioned properly for each user's eyes. This adjustment was achieved by turning the lateral adjuster to fix the separation between the participant's eyes (inter-pupillary distance). The HMD was kept firmly in place by strapping it tightly to the participant's face.

In the large stereo screen condition, the participants were standing in front of the large, high-resolution display. Displacement and rotation depend on the decision points shown in the virtual environment. Each participant was instructed about how to use the gamepad, the HMD, and the polarized glasses. The participant was also urged to pay attention at each stage of the exposure. Each participant was instructed to remember the route in order to find a way out of the maze.

After ending the session, the participants answered a questionnaire on the interaction with the system, 3D sensations, and satisfaction (PQ). The questions of the questionnaire are shown in Table 2.

\section{Results}

This section presents the analysis of the data collected from this study. Data normality was checked and the pertinent statistical tests were carried out based on those results. The Shapiro-Wilk and Anderson-Darling are inferential tests that were used to check data normality. Since the tests reported that our data did not fit the normal distribution, non-parametric statistical tests (the Mann-Whitney U test) were applied for the Likert questions to determine whether or not there were statistically significant differences for our questionnaire (Table 2). There were two groups: one group used the HMD and the other group used the large stereo screen. These two groups were also divided into two different populations, those participants who had stereopsis and those participants who 
Table 2. Questionnaire on the interaction, 3D-sensations, and satisfaction. The questionnaire used a Likert scale [from 1 to 5 ( 1 being 'none' or extremely low and 5 being 'very high')].

\begin{tabular}{l|l}
\hline \# QI & Interaction \\
\hline QI1 & How natural was the mechanism that controlled movement through the environment? \\
\hline QI2 & How natural did your interactions with the 3D environment seem? \\
\hline QI3 & $\begin{array}{l}\text { How well could you concentrate on the required tasks rather than on the mechanisms } \\
\text { used to perform those tasks? }\end{array}$ \\
\hline QI4 & The environment was easy to use \\
\hline \# QE & Virtual Environment and 3D-sensations \\
\hline QE1 & How involved were you in the 3D virtual environment experience? \\
\hline QE2 & $\begin{array}{l}\text { How much did your experiences in the virtual environment seem consistent with your } \\
\text { real-world experiences? }\end{array}$ \\
\hline QE3 & How closely were you able to examine objects? \\
\hline QE4 & How quickly did you adjust to the 3D virtual environment experience? \\
\hline QE5 & At times it seems to me that objects have depth? \\
\hline QE6 & My 3D experience compared to others previous 3D experiences has been ... \\
\hline \# QS & Satisfaction \\
\hline QS1 & To what degree did you feel general discomfort during or at the end of the task? \\
\hline QS2 & $\begin{array}{l}\text { In general, rate the experience of movement and interaction with the virtual } \\
\text { environment }\end{array}$ \\
\hline QS3 & Rate your visualization experience from 1-5 (1-least satisfying) \\
\hline
\end{tabular}

did not have stereopsis. The data from the study were analyzed using the statistical open source toolkit R (http://www.r-project.org) with the R-Studio IDE (http://www. rstudio.com).

The results of our questionnaire were grouped by Interaction, 3D Sensations, and Satisfaction. The results are shown in Tables 3, 4, 5, 6, 7 and 8 .

\subsection{Interaction Outcomes}

As Tables 3 and 4 show, no statistically significant differences were found in the QI2QI4 questions between the HMD condition and the large stereo screen condition. The participants thought that the interaction with the 3D environment seemed natural (QI2). The users were concentrated on the assigned task rather than on the mechanisms used to perform it (QI3). The participants did not perceive significant differences for ease of use (QI4). However, there was a statistically significant difference in QI1 in favor of the HMD. In Q1, the participants perceived the mechanism, which controlled movement through the environment, to be more natural. These results were obtained for the two groups of participants (stereopsis vs. no stereopsis).

For the HMD condition and the two population groups (stereopsis vs. no stereopsis), no statistically significant differences were found in the QI1-QI4 questions. The same result was obtained for the large stereo screen condition and the two population groups. 
Table 3. Means and Standard deviations, Mann-Whitney $U$ test analysis, and $r$ effect size between the HMD condition and the large stereo screen condition of those who did not have stereopsis for the questions about interaction. The ${ }^{* *}$ indicates statistically significant differences.

\begin{tabular}{l|l|l|l|l|l|l}
\hline \# Q & HMD & Large stereo screen & $U$ & $Z$ & $p$ & $r$ \\
\hline QI1 & $4.38 \pm 0.52$ & $1.14 \pm 0.38$ & 56.0 & 3.426 & $<0.001 * *$ & 0.885 \\
\hline QI2 & $4.88 \pm 0.35$ & $4.43 \pm 1.13$ & 33.0 & 0.829 & 0.446 & 0.214 \\
\hline QI3 & $4.00 \pm 0.93$ & $3.86 \pm 0.69$ & 30.5 & 0.308 & 0.962 & 0.079 \\
\hline QI4 & $4.13 \pm 0.64$ & $3.14 \pm 1.22$ & 41.5 & 1.662 & 0.101 & 0.429 \\
\hline
\end{tabular}

Table 4. Means and Standard deviations, Mann-Whitney U test analysis, and $r$ effect size between the HMD condition and the large stereo screen condition of those who had stereopsis for the questions about interaction. The ** indicates statistically significant differences.

\begin{tabular}{l|l|l|l|l|l|l}
\hline$\#$ Q & HMD & Large stereo screen & $U$ & $\mathrm{Z}$ & $p$ & $r$ \\
\hline QI1 & $4.00 \pm 0.93$ & $1.14 \pm 0.35$ & 481.0 & 5.906 & $<0.001 * *$ & 0.890 \\
\hline QI2 & $4.64 \pm 0.73$ & $4.36 \pm 0.66$ & 307.0 & 1.757 & 0.101 & 0.265 \\
\hline QI3 & $4.09 \pm 0.68$ & $3.59 \pm 0.91$ & 319.0 & 1.952 & 0.055 & 0.294 \\
\hline QI4 & $4.00 \pm 0.93$ & $3.77 \pm 1.31$ & 255.5 & 0.332 & 0.747 & 0.050 \\
\hline
\end{tabular}

\subsection{D-Sensation Outcomes}

To determine the outcomes for 3D sensations, the participants answered questions QE1-QE6 after their exposure to the virtual environment in two conditions (HMD vs. large stereo screen). Statistically significant differences were found in all six questions in favor of the HMD. These statistically significant differences can be observed for the group of participants who did not have stereopsis (Table 5 and Fig. 6) and those who had (Table 6 and Fig. 6). Overall, the HMD allowed the participants to feel a more enhanced experience than the large stereo screen for the two groups (stereopsis vs. no stereopsis).

For the HMD condition and the two groups of population (stereopsis vs. no stereopsis), no statistically significant differences were found in the QE1-QE6 questions. For the large stereo screen condition, no statistically significant differences were found for any of the questions, except for QE3 in favor of the participants who had stereopsis $(U=32, Z=-2.687, p=0.011, r=0.499)$. Although the means of the two groups for QE3 are low, the participants who had stereopsis were able to closely examine objects to a significantly greater extent than the participants who did not have stereopsis. Moreover, the participants who had stereopsis in the large stereo condition scored higher in all the questions (except QE1) than those who did not have stereopsis.

In QS1, there were no statistically significant differences between the two conditions for the participants who did not have stereopsis regarding general discomfort during or at the end of the session (see Table 7). However, in QS1, there was a statistically significant difference between the two conditions and for the participants who had stereopsis (see Table 8). The values of the means for the two groups show that the participants who had stereopsis felt greater general discomfort with the HMD. 
Table 5. Means and Standard deviations, Mann-Whitney $U$ test analysis, and $r$ effect size between the HMD condition and the large stereo screen condition of those who did not have stereopsis for the questions about 3D sensations. The $* *$ indicates statistically significant differences.

\begin{tabular}{l|l|l|l|l|l|l}
\hline \# Q & HMD & Large stereo screen & $U$ & $Z$ & $p$ & $r$ \\
\hline QE1 & $4.63 \pm 0.52$ & $3.57 \pm 0.54$ & 50.0 & 2.750 & $0.009^{* *}$ & 0.710 \\
\hline QE2 & $3.62 \pm 0.74$ & $1.14 \pm 0.38$ & 56.0 & 3.392 & $<0.001^{* *}$ & 0.876 \\
\hline QE3 & $3.88 \pm 0.84$ & $1.14 \pm 0.38$ & 56.0 & 3.376 & $<0.001^{* *}$ & 0.872 \\
\hline QE4 & $4.38 \pm 0.74$ & $3.43 \pm 0.54$ & 46.5 & 2.277 & $0.034^{* *}$ & 0.588 \\
\hline QE5 & $4.00 \pm 0.54$ & $2.29 \pm 0.76$ & 54.5 & 3.210 & $<0.001^{* *}$ & 0.829 \\
\hline QE6 & $3.75 \pm 0.89$ & $1.71 \pm 1.11$ & 50.5 & 2.726 & $0.008^{* *}$ & 0.704 \\
\hline
\end{tabular}

Table 6. Means and Standard deviations, Mann-Whitney $U$ test analysis, and $r$ effect size between the HMD condition and the large stereo screen condition of those who had stereopsis for the questions about 3D sensations. The ** indicates statistically significant differences.

\begin{tabular}{l|l|l|l|l|l|l}
\hline \# Q & HMD & Large stereo screen & $U$ & $Z$ & $p$ & $r$ \\
\hline QE1 & $4.18 \pm 0.96$ & $3.50 \pm 1.01$ & 338.0 & 2.365 & $0.018^{* *}$ & 0.357 \\
\hline QE2 & $3.68 \pm 1.13$ & $1.18 \pm 0.40$ & 465.0 & 5.510 & $<0.001^{* *}$ & 0.831 \\
\hline QE3 & $3.96 \pm 0.84$ & $1.73 \pm 0.46$ & 476.0 & 5.712 & $<0.001^{* *}$ & 0.861 \\
\hline QE4 & $4.46 \pm 0.51$ & $3.50 \pm 0.91$ & 385.0 & 3.590 & $<0.001^{* *}$ & 0.541 \\
\hline QE5 & $4.14 \pm 0.77$ & $2.64 \pm 1.18$ & 405.0 & 3.964 & $<0.001^{* *}$ & 0.598 \\
\hline QE6 & $3.96 \pm 1.09$ & $1.86 \pm 1.21$ & 423.5 & 4.398 & $<0.001^{* *}$ & 0.663 \\
\hline
\end{tabular}

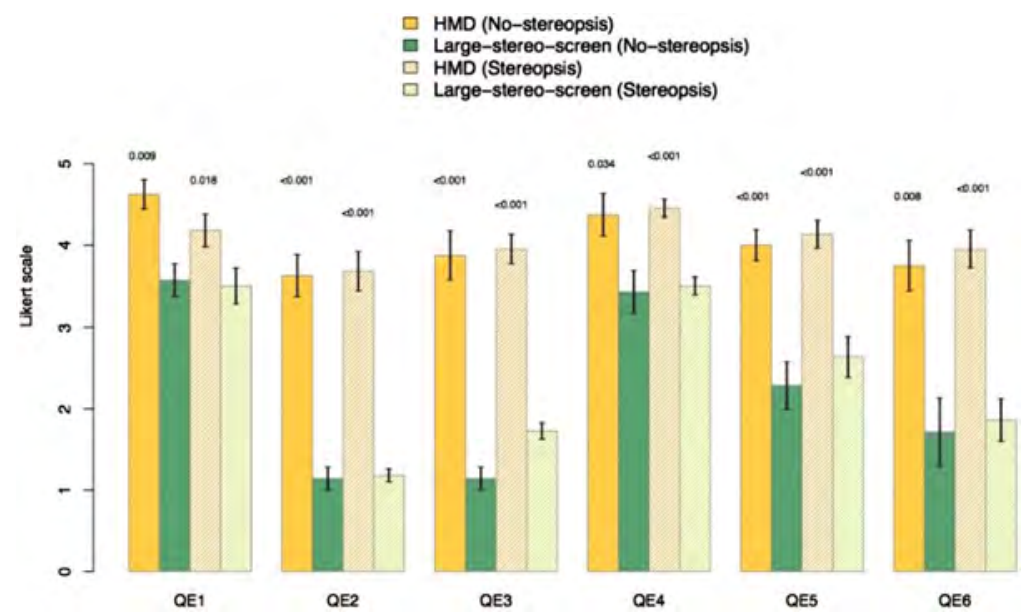

Fig. 6. Participants who had stereopsis and participants who did not have stereopsis (HMD vs. large stereo screen). Barplot and error bars for QE1-QE6 questions. Confidence interval of 95\%. Statistically significant differences are found in all questions. Satisfaction outcomes 
Table 7. Means and Standard deviations, Mann-Whitney $U$ test analysis, and $r$ effect size between the HMD condition and the large stereo screen condition of those who did not have stereopsis for the questions about satisfaction. The ** indicates statistically significant differences.

\begin{tabular}{l|l|l|l|l|l|l}
\hline \# Q & HMD & Large stereo screen & $U$ & $Z$ & $p$ & $r$ \\
\hline QS1 & $1.50 \pm 0.53$ & $1.14 \pm 0.37$ & 38.0 & 1.414 & 0.282 & 0.365 \\
\hline QS2 & $3.75 \pm 0.46$ & $1.43 \pm 0.53$ & 56.0 & 3.395 & $<0.001^{* *}$ & 0.877 \\
\hline QS3 & $4.38 \pm 0.52$ & $3.57 \pm 0.53$ & 46.0 & 2.372 & $0.039^{* *}$ & 0.612 \\
\hline
\end{tabular}

Table 8. Means and Standard deviations, Mann-Whitney U test analysis, and $r$ effect size between the HMD condition and the large stereo screen condition of those who had stereopsis for the questions about satisfaction. The ** indicates statistically significant differences.

\begin{tabular}{l|l|l|l|l|l|l}
\hline \# Q & HMD & Large stereo screen & $U$ & $Z$ & $p$ & $r$ \\
\hline QS1 & $1.55 \pm 0.80$ & $1.14 \pm 0.35$ & 311.0 & 2.084 & $0.055^{* *}$ & 0.314 \\
\hline QS2 & $4.09 \pm 0.81$ & $3.32 \pm 0.48$ & 369.0 & 3.241 & $<0.001^{* *}$ & 0.489 \\
\hline QS3 & $4.45 \pm 0.74$ & $3.59 \pm 0.85$ & 371.5 & 3.209 & $<0.001^{* *}$ & 0.484 \\
\hline
\end{tabular}

In QS2 and QS3, the results show that there were statistically significant differences between the two conditions in favor of the HMD (see Tables 7 and 8). This means that, in general, the participants had a more satisfying experience using the HMD.

For the HMD condition and the two population groups (stereopsis vs. no stereopsis), no statistically significant differences were found in the QS1-QS3 questions. For the large stereo screen condition, no statistically significant differences were found for any of the questions, except for QS2 in favor of the participants who had stereopsis. This result for QS2 implies that the participants who had stereopsis rated the experience of movement and interaction with the virtual environment significantly higher than the participants who did not have stereopsis. When comparing the HMD column of Tables 7 and 8 (HMD condition, no stereopsis vs. stereopsis), the participants who had stereopsis scored higher on all the questions than the participants who did not have stereopsis. The results for the comparison of the large stereo screen column were similar.

\subsection{Task Outcomes}

To determine the performance on the task, we calculated the following: the time for completion of the task in seconds (Time), the number of head turns by the participant performed at intersections (Headings), the number of attempts made to successfully complete the path (Attempts), and the score (Score). The Score was obtained by adding the number of correct directions chosen in each of the five attempts established to complete the path. We defined ten points per attempt and a maximum Score of fifty points. Specifically, the Score variable was obtained as follows. Each participant had five attempts to reach the end of the maze. If the participant reached the end of the maze on the first attempt, the task ended. If a participant chose the wrong direction at an intersection, the participant automatically returned to the starting point and made another attempt. If the participant went through all five attempts, the task ended. 
The participants received a point for each correct choice of an intersection at each attempt. There were 10 intersections in total. The participants received 10 points for each attempt that they did not have to complete. Therefore, if the participants reached the end of the maze on the first attempt, they received 50 points (10 for the first attempt, and 40 for the 4 attempts that they did not have to complete).

The results for the participants with stereopsis and those without stereopsis using the HMD condition are shown in Table 9. Table 10 shows the results considering gender. The results for the participants with stereopsis and those without stereopsis using the large stereo condition are shown in Table 11. Table 12 shows the results taking gender into account. For the two conditions considered independently, the results show that there were no statistically significant differences for the performance on the task between the participants with stereopsis and the participants without stereopsis. The performance on the task was also independent for gender.

Table 9. Means and Standard deviations, Mann-Whitney U test analysis, and $\mathrm{r}$ effect size for the HMD condition and between the participants without stereopsis and those with stereopsis.

\begin{tabular}{l|c|c|r|r|l|l}
\hline & No-stereopsis & \multicolumn{1}{l|}{ Stereopsis } & $U$ & \multicolumn{1}{l}{$Z$} & $p$ & $r$ \\
\hline Score & $48.0 \pm 2.88$ & $47.68 \pm 3.23$ & 97.0 & 0.459 & 0.667 & 0.084 \\
\hline Attempts & $1.62 \pm 0.74$ & $2.05 \pm 1.09$ & 70.0 & -0.898 & 0.395 & 0.164 \\
\hline Time & $350.5 \pm 191.50$ & $295.89 \pm 109.63$ & 106.0 & 0.844 & 0.414 & 0.154 \\
\hline
\end{tabular}

Table 10. Means and Standard deviations, Kruskal-Wallis test analysis for the HMD condition and for gender. Atte. = Attempts.

\begin{tabular}{|c|c|c|c|c|c|c|c|}
\hline & \multicolumn{2}{|l|}{ No-stereopsis } & \multicolumn{2}{|l|}{ Stereopsis } & \multirow[t]{2}{*}{$\chi^{2}$} & \multirow[t]{2}{*}{$d f$} & \multirow[t]{2}{*}{$p$} \\
\hline & Men & Women & Men & Women & & & \\
\hline Score & $48.00 \pm 3.16$ & $48.00 \pm 2.83$ & $48.83 \pm 2.37$ & $46.30 \pm 3.68$ & 0.21 & 1 & 0.646 \\
\hline Atte. & $1.67 \pm 0.82$ & $1.50 \pm 0.71$ & $2.00 \pm 1.34$ & $2.10 \pm 0.74$ & 0.77 & 1 & 0.379 \\
\hline Time & $364.1 \pm 218.40$ & $309.70 \pm 116.6$ & $296.30 \pm 129.40$ & $295.4 \pm 87.0$ & 0.71 & 1 & 0.399 \\
\hline
\end{tabular}

Table 11. Means and Standard deviations, Mann-Whitney $U$ test analysis, and $r$ effect size for the large stereo condition for the participants without stereopsis and those with stereopsis.

\begin{tabular}{l|c|c|l|r|l|l}
\hline & No-stereopsis & Stereopsis & $U$ & \multicolumn{1}{l}{$Z$} & \multicolumn{1}{l}{$l$} & $r$ \\
\hline Score & $41.43 \pm 9.41$ & $38.09 \pm 11.36$ & 91.0 & 0.729 & 0.484 & 0.135 \\
\hline Attempts & $2.14 \pm 1.46$ & $2.95 \pm 1.76$ & 58.5 & -0.983 & 0.373 & 0.183 \\
\hline Time & $114.5 \pm 31.56$ & $137.53 \pm 59.34$ & 67.0 & -0.510 & 0.636 & 0.095 \\
\hline
\end{tabular}


Table 12. Means and Standard deviations, Kruskal-Wallis test analysis for the large stereo condition and for gender. Atte. $=$ Attempts

\begin{tabular}{l|l|l|l|l|l|l|l}
\hline & No-stereopsis & \multicolumn{2}{|l|}{ Stereopsis } & \multirow{2}{*}{$\chi^{2}$} & $d f$ & $p$ \\
\cline { 1 - 4 } & Men & Women & Men & Women & & & \\
\hline Score & $41.00 \pm 10.82$ & $41.75 \pm 9.95$ & $40.14 \pm 9.94$ & $43.50 \pm 13.44$ & 0.37 & 1 & 0.541 \\
\hline Atte. & $1.67 \pm 0.58$ & $2.50 \pm 1.91$ & $2.79 \pm 1.72$ & $3.25 \pm 1.91$ & 0.31 & 1 & 0.579 \\
\hline Time & $99.69 \pm 15.69$ & $125.59 \pm 38.01$ & $130.71 \pm 59.73$ & $149.47 \pm 60.66$ & 1.23 & 1 & 0.268 \\
\hline
\end{tabular}

Table 13. Multifactorial ANOVA test for the Headings variable, $\mathrm{N}=30$.

\begin{tabular}{l|l|l|l}
\hline Factor & $F$ & $p$ & Effect size $\left(\eta^{2}\right)$ \\
\hline Gender & 0.009 & 0.924 & $<0.01$ \\
\hline Group (no stereopsis/stereopsis) & 0.003 & 0.961 & $<0.01$ \\
\hline Gender:Group & 0.046 & 0.832 & $<0.01$ \\
\hline
\end{tabular}

For the HMD condition, Table 13 shows the results for the Headings variable (head turnings) taking into account gender and group (no stereopsis vs. stereopsis). The results show that there were no statistically significant differences for gender and group.

When the Score variable for the group who did not have stereopsis is analyzed and the HMD $(48.00 \pm 2.88)$ and large stereo screen $(41.43 \pm 9.41)$ conditions are compared, there were no statistically significant differences $(U=38.5, Z=1.318$, $\mathrm{p}=0.210, \mathrm{r}=0.340)$. When the Score variable for the group who had stereopsis is analyzed and the HMD $(47.68 \pm 3.23)$ and large stereo screen $(38.09 \pm 11.36)$ conditions are compared, there were statistically significant differences $(\mathrm{U}=354$, $\mathrm{Z}=2.725, \mathrm{p}=0.006^{* *}, \mathrm{r}=0.411$ ) in favor of the HMD condition.

\section{Discussions}

As mentioned in the background section, HMDs have already been compared with different visualization systems. In this paper, we have compared a HMD (Oculus Rift DK2) with a partially immersive VR system. Previous works have compared the Oculus Rift with a fully immersive VR system [13] and with a non-immersive VR system [15]. Our results are in line with these works. Although other works have suggested that a large projection screen may be an effective substitute for a HMD [27], our results indicate that participants had a better 3D experience using a HMD than using a large stereo screen. Juan \& Perez [17] compared a HMD and a CAVE and observed that the CAVE induced a significantly higher level of presence. The features of their HMD were: $800 \times 600$ and $40^{\circ}$ FOV. The features of the current HMDs are significantly better. We used a HMD with $960 \times 1080$ and $100^{\circ} \mathrm{FOV}$. Another aspect to consider is the inclusion in the system of head tracking. The motion parallax cue plays an important role in stereoscopy. In a fair comparison, the projected stereoscopic display should have head tracking. From our results (QI1 and QS2), non-inclusion of head tracking has negatively affected the results for our large stereo screen condition. 
In any case, considering our work and previous works, it is possible to conclude that current HMDs offer advantages over basic, partially, or fully immersive VR systems.

Also mentioned in the introduction section, the study was motivated by the observation of students who did not have stereopsis and did not have depth perception with other VR devices (e.g., CAVE, a large stereo screen, or autostereoscopic displays). However, those same students did have the sensation of depth using the Oculus Rift. Our study corroborated our main hypothesis that current HMDs allow users with stereopsis problems to have the illusion of depth perception. Our explanation for this is that the field of view of current HMDs is much more similar to the human eye than other VR devices or systems. The inclusion of head tracking and a low latency are also very important. Nearly all of the current HMDs include head tracking. As Carmack [28] indicated that "The latency between the physical movement of a user's head and updated photons from a HMD reaching their eyes is one of the most critical factors in providing a high quality experience". Thus, all the new features of current HMDs allow the users to perceive the virtual environment similarly to the way they perceive reality, and, therefore, they feel similar sensations. Stereo blind individuals rely more heavily on motion based cues for depth. Therefore, the 3D experience could largely be influenced by the head tracking. Our argument that the head tracking largely influences the 3D experience was shared by one of the participants without stereopsis. This participant was a computer graphics $\mathrm{PhD}$ student, and he explained his experience in an interview after 3 months of his participation in our study. The participant was not able to identify any of the figures that appear in the Lang 1 Stereotest. He did not perceive 3D with an autostereoscopic screen, or with the large stereo screen used in our study, or in the 3D cinemas. However, for the first time in his life, he did experience the feeling of depth with a VR environment using the Oculus Rift. With our virtual maze, he could perceive that the virtual elements were at his side and he could notice the distance they were from. His personal opinion was that the changes in perspective while moving his head enabled him to have that 3D feeling. After this first 3D experience, he tested other stereoscopic devices and he has only been able to appreciate 3D with HMDs that include head tracking. This participant added that when using HMDs that do not include head tracking, instead of perceiving 3D, he suffered from cybersickness. He also experienced cybersickness with the Oculus Rift and with environments that do not allow navigation using head turns. These $3 \mathrm{D}$ experiences have not changed the way he perceives objects in the real world. Other statements expressed by other participants without stereopsis during the experience were as follows: 'Oh my God, I can perceive $3 \mathrm{D}$ for the first time in my life with this VR device'. This reaction was in line with that reported by the participants in the study carried out by Ding \& Levi [9], "depth 'popped out' in daily life, and I enjoyed 3D movies for the first time".

Previous works have used VR for training adults who were stereo blind or stereodeficient [12]. After the training, some of those participants recovered or acquired stereopsis. Our work tested the same virtual environment with two different visualization systems (HMD vs. a large stereo screen) and with people with stereopsis and without stereopsis. From the results, the HMD allowed the participants to feel a richer 3D experience than the large stereo screen for both groups (stereopsis vs. no stereopsis). This also indicates that full stereopsis may not be necessary for rich 3D experiences. The performance on the task for the HMD was independent from the 
participants' condition (stereopsis vs. no stereopsis) and gender. Therefore, our work and previous works are complementary and their union opens up new possibilities for people with stereoblindness or stereodeficiency. We believe that the use of HMDs in training people for recovering or acquiring stereopsis could have implications for the recovery of visual function in real life. Several studies have indicated that between $5 \%$ and $10 \%$ of the population do not have stereoscopic vision [23, 25]. This percentage can be as high as 34\% in older subjects [26]. Therefore, current HMDs could help this population to experience depth perception using VR. As mentioned in the background section, Bridgeman [8], with stereo-deficiency, acquired stereopsis when watching a $3 \mathrm{D}$ movie. A current HMD has been used for watching 3D movies as an observer or as an actor [29]. Oculus Story Studios (https://storystudio.oculus.com/en-us) made their first two movies, Lost (2015) and Henry (2016). The possibility of watching movies in $3 \mathrm{D}$ as an observer or as an actor is interesting for people with stereopsis, but it also opens up a new possibility for people with stereopsis problems that could be explored.

In this study, we have used a gamepad for the interaction. However, other devices or types of interaction can also be used, e.g., using touch motion controllers, which can be combined with the Oculus Rift CV1. Another possibility is to use VR Manus gloves, or to use Leap Motion for gesture interaction. Leap Motion can be attached to the HMD, allowing interaction with the user's hands.

Even though current HMDs have several benefits, they also have some drawbacks. One of them is the cybersickness that they may induce. As Davis et al. [18] indicated, the more realistic the environment with higher levels of visual flow, the greater the chance of inducing cybersickness. Other works have also studied cybersickness. For example, Sharples et al. [30] studied VR induced symptoms and effects comparing a HMD, a desktop, a projection screen (smaller than ours), and a reality theatre. The participants using the HMD and the projection screen experienced a significant increase in symptoms pre-post exposure for oculomotor, disorientation, and total scored. Moreover, the participants using the HMD also reported a significant increase in nausea. We have not carried out a formal study about cybersickness, but the data for the SQ1 question (To what degree did you feel general discomfort during or at the end of the task?) indicates that the participants who had stereopsis scored significantly higher on SQ1 using the HMD than using the large stereo screen condition. Taking into account the differences, our observations are in line with the conclusions obtained by Sharples et al. [30]. Recent studies indicate that the Oculus Rift induces motion sickness [31]. However, further studies are needed to determine whether this or other current HMDs induce more cybersickness than other VR systems, and comparisons between them should also be made. Another drawback is that cables must be connected to the computer. Therefore, wireless HMDs (e.g., Samsung Gear VR, Google Cardboard or HoloLens) that offer freedom of movement could also be considered.

\section{Conclusion}

We have compared two different visualization systems: a partially immersive large stereo screen, and a fully immersive HMD. The study involved participants who had stereopsis and participants who did not have stereopsis. To our knowledge, this is the 
first comparison involving those two different visualization systems and those two population groups. The HMD has provided a significantly better VR experience than the large stereo screen. Users that have stereopsis problems and cannot perceive 3D when looking at the Lang 1 Stereo test or using other VR systems (CAVE, large stereo screens, or autostereoscopic displays) do have the sensation of depth when using the HMD. Therefore, our findings indicate that people without stereopsis may benefit from a 3D experience with current HMDs.

As future work regarding the perceptions of people that do not have stereopsis when using current HMDs, a study could be carried out to determine the weight of the different aspects that influence stereoscopy (especially, motion parallax). The Oculus Rift, other HMDs, or other 3D-display technologies could be used to design VR environments for training and to facilitate recovery of stereo vision by people with stereo-deficiencies. We hope to help people who are afflicted with stereo-deficiencies to have rich $3 \mathrm{D}$ experiences in VR with the work and ideas presented here.

\section{Acknowledgments.}

- This work was mainly funded by the Spanish Ministry of Economy and Competitiveness (MINECO) through the CHILDMNEMOS project (TIN2012-37381-C02-01) and cofinanced by the European Regional Development Fund (FEDER).

- Other financial support was received from the Government of the Republic of Ecuador through the Scholarship Program of the Secretary of Higher Education, Science, Technology and Innovation (SENESCYT), the Conselleria d'Educació, Investigació, Cultura i Esport through the grant for consolidable research groups (2017-2018), the Government of Aragon (Department of Industry and Innovation), and the European Social Fund for Aragon.

- We would like to thank the following for their contributions:

- David Rodríguez Andrés, Mauricio Loachamín Valencia, and Juan Fernando Martín for their help.

- DSIC and ASIC for allowing us to use its facilities during the testing phase, especially to Vicente Blasco and Manuel Jiménez.

- The users who participated in the study.

- The reviewers for their valuable comments.

\section{References}

1. Howard, I.P., Rogers, B.J.: Binocular Vision and Stereopsis. Oxford University Press, New York (1995)

2. Howard, I.P., Rogers, B.J.: Perceiving in depth, vol. 3. Oxford University Press, New York (2012)

3. Barry, S.R.: Fixing My Gaze: A Scientist's Journey Into Seeing in Three Dimensions. Basic Books, New York (2009)

4. LaViola, J.L.: A discussion of cybersickness in virtual environments. ACM SIGCHI Bull. 32 (January), 47-56 (2000)

5. Dionisio, J.D.N., Burns III, W.G., Gilbert, R.: 3D virtual worlds and the metaverse: current status and future possibilities. ACM Comput. Surv. 45(3), 34 (2013)

6. Muhanna, M.A.: Virtual reality and the CAVE: taxonomy, interaction challenges and research directions. J. King Saud Univ. Comput. Inf. Sci. 27, 344-361 (2015) 
7. Sutherland, I.E.: A head-mounted, three-dimensional display. In: The Fall Joint Computer Conference, pp. 757-764 (1968)

8. Bridgeman, B.: Restoring adult stereopsis: a vision researcher's personal experience. Optom. Vis. Sci. 91, e135-e139 (2014)

9. Ding, J., Levi, D.M.: Recovery of stereopsis through perceptual learning in human adults with abnormal binocular vision. Proc. Natl. Acad. Sci. 108(37), E733-E741 (2011). USA

10. Astle, A.T., McGraw, P.V., Webb, B.S.: Recovery of stereo acuity in adults with amblyopia. BMJ Case Rep. 2011: bcr0720103143 (2011)

11. Xi, J., Jia, W.-L., Feng, L.-X., Lu, Z.-L., Huang, C.-B.: Perceptual learning improves stereoacuity in amblyopia. Invest. Ophthalmol. Vis. Sci. 55, 2384-2391 (2014)

12. Vedamurthy, I., Knill, D.C., Huang, S.J., Yung, A., Ding, J., Kwon, O.-S., Bavelier, D., Levi, D.M.: Recovering stereo vision by squashing virtual bugs in a virtual reality environment. Philos. Trans. R. Soc. Lond. B Biol. Sci. 371(1697), 20150264 (2016)

13. Young, M.K., Gaylor, G., B., Andrus, S.M., Bodenheimer, B.: A comparison of two cost-differentiated virtual reality systems for perception and action tasks. In: The ACM Symposium on Applied Perception, pp. 83-90 (2014)

14. Buń, P., Górski, F., Wichniarek, R., Kuczko, W., Hamrol, A., Zawadzki, P.: Application of professional and low-cost head mounted devices in immersive educational application. Procedia Comput. Sci. 75, 173-181 (2015)

15. Tan, C.T., Leong, T.W., Shen, S., Dubravs, C., Si, C.: Exploring gameplay experiences on the oculus rift. In: CHI PLAY 2015, pp. 253-263 (2015)

16. Gutiérrez-Maldonado, J., Ferrer-García, M., Plasanjuanelo, J., Andrés-Pueyo, A., Talarn-Caparrós, A.: Virtual reality to train diagnostic skills in eating disorders. comparison of two low cost systems. Stud. Health Tech. Inf. 219, 75-81 (2015)

17. Juan, M.C., Pérez, D.: Comparison of the Levels of presence and anxiety in an acrophobic environment viewed via HMD or CAVE. Presence Teleoper. Virtual Environ. 18(3), 232248 (2009)

18. Davis, S., Nesbitt, K., Nalivaiko, E.: Comparing the onset of cybersickness using the Oculus Rift and two virtual roller coasters. In: the 11th Australasian Conference on Interactive Entertainment, vol. 167, pp. 3-14 (2015)

19. Arias, N., Méndez, M., Arias, J.L.: Brain networks underlying navigation in the Cincinnati water maze with external and internal cues. Neurosci. Lett. 576, 68-72 (2014)

20. Cárdenas-Delgado, S., Méndez-López, M., Juan, M.C., Pérez-Hernández, E., Lluch, J., Vivó, R.: Using a Virtual Maze Task to assess spatial short-term memory in adults. In: International Conference on Computer Graphics Theory and Applications, pp. 46-57 (2017)

21. Dodgson, N. A.: Variation and extrema of human interpupillary distance. In: SPIE 5291, Stereoscopic Displays and Virtual Reality Systems XI, pp. 36-46 (2004)

22. Lang, J.: A new stereotest. J. Pediatr. Ophthalmol. Strabismus 20(2), 72-74 (1983)

23. Brown, S., Weih, L., Mukesh, N., McCarty, C., Taylor, H.: Assessment of adult stereopsis using the Lang 1 Stereotest: a pilot study. Binocular Vis. Strabismus Q. 16(2), 91-98 (2001)

24. Witmer, B.G., Singer, M.J.: Measuring presence in virtual environments: a presence questionnaire. Presence Teleoper. Virtual 7(3), 225-240 (1998)

25. Castanes, M.S.: Major review: The underutilization of vision screening (for amblyopia, optical anomalies and strabismus) among preschool age children. Binocular Vis. Strabismus Q. 18(4), 217-232 (2003)

26. Zaroff, C.M., Knutelska, M., Frumkes, T.E.: Variation in stereoacuity: normative description, fixation disparity, and the roles of aging and gender. Invest. Ophthalmol. Vis. Sci. 44, 891-900 (2003) 
27. Patrick, E., Cosgrove, D., Slavkovic, A., Rode, J.A., Verratti, T., Chiselko, G.: Using a large projection screen as an alternative to head-mounted displays for virtual environments. In: SIGCHI Conference on Human Factors in Computing Systems, pp. 478-485 (2000)

28. Carmack, J.: John Carmack's Latency mitigation strategies (2013). http://www.pcgamesn. com/virtual-reality-john-carma. Accessed 20 Jan 2017

29. van den Boom, A.A.L.F.M., Stupar-Rutenfrans, S., Bastiaens, O., van Gisbergen, M.S.: Observe or participate: the effect of point-of-view on presence and enjoyment in 360 degree movies for head mounted displays. In: the European Conference on Ambient Intelligence (2015). http://ceur-ws.org/Vol-1528/paper13.pdf. Accessed 20 Jan 2017

30. Sharples, S., Cobb, S., Moody, A., Wilson, J.R.: Virtual reality induced symptoms and effects (VRISE): comparison of head mounted display (HMD), desktop and projection display systems. Displays 29(2), 58-69 (2008)

31. Munafo, J., Diedrick, M., Stoffregen, T.A.: The virtual reality head-mounted display Oculus Rift induces motion sickness and is sexist in its effects. Exp. Brain Res. 235, 889-901 (2016). 